\title{
Cardioprotective Effects of Voluntary Exercise in a Rat Model: Role of Matrix Metalloproteinase-2
}

\author{
Anikó Pósa, ${ }^{1}$ Renáta Szabó, ${ }_{1}^{1}$ Krisztina Kupai, ${ }^{1}$ Zoltán Baráth, ${ }^{2}$ \\ Zita Szalai, ${ }^{1}$ Anett Csonka, ${ }^{1}$ Médea Veszelka, ${ }^{1}$ Mariann Gyöngyösi, ${ }^{3}$ Zsolt Radák, ${ }^{4}$ \\ Rudolf Ménesi, ${ }^{1}$ Imre Pávó, ${ }^{1}$ Anikó Magyariné Berkó, ${ }^{1}$ and Csaba Varga ${ }^{1}$

\begin{abstract}
${ }^{1}$ Department of Physiology, Anatomy and Neuroscience, University of Szeged, Kozep Fasor 52, Szeged 6726, Hungary
${ }^{3}$ Department of Cardiology, Medical University of Vienna, Waehringer Guertel 18-20, 1090 Vienna, Austria

${ }^{4}$ Institute of Sport Science, Faculty of Physical Education and Sport Science, Semmelweis University, Alkotas Ucta 44,
\end{abstract} \\ ${ }^{2}$ Faculty of Dentistry and Department of Orthodontics and Pediatric Dentistry, University of Szeged, Szeged 6720, Hungary \\ Budapest 1123, Hungary
}

Correspondence should be addressed to Anikó Pósa; paniko@bio.u-szeged.hu

Received 12 September 2014; Accepted 24 November 2014

Academic Editor: P. Venditti

Copyright (C) 2015 Anikó Pósa et al. This is an open access article distributed under the Creative Commons Attribution License, which permits unrestricted use, distribution, and reproduction in any medium, provided the original work is properly cited.

\begin{abstract}
Background. Regular exercise at moderate intensity reduces cardiovascular risks. Matrix metalloproteinases (MMPs) play a major role in cardiac remodeling, facilitating physiological adaptation to exercise. The aim of this study was to examine the influence of voluntary physical exercise on the MMP-2 enzyme activity and to investigate the cardiac performance by measurement of angina susceptibility of the heart, the basal blood pressure, the surviving aorta ring contraction, and the cardiac infarct size after I/Rinduced injury. Methods. Male Wistar rats were divided into control and exercising groups. After a 6-week period, the serum level of MMP-2, basal blood pressure, cardiac angina susceptibility (the ST segment depression provoked by epinephrine and $30 \mathrm{~s}$ later phentolamine), AVP-induced heart perfusion and aorta ring contraction, infarct size following $30 \mathrm{~min}$ ischemia and $120 \mathrm{~min}$ reperfusion, and coronary effluent MMP-2 activity were measured. Results. Voluntary wheel-running exercise decreased both the sera $(64 \mathrm{kDa}$ and $72 \mathrm{kDa})$ and the coronary effluent $(64 \mathrm{kDa}) \mathrm{MMP}-2$ level, reduced the development of ST depression, improved the isolated heart perfusion, and decreased the ratio of infarct size. Conclusion. 6 weeks of voluntary exercise training preserved the heart against cardiac injury. This protective mechanism might be associated with the decreased activity of MMP-2.
\end{abstract}

\section{Introduction}

Regular physical exercise has been shown to reduce many cardiovascular risk factors and is associated with a number of cardiovascular benefits. The reduced levels of cardiovascular mortality and cardiac ischemic events in patients who participate in regular exercise training are mainly due to the control of cardiovascular risk factors (e.g., high blood pressure and obesity) $[1,2]$.

Although regular exercise training has been confirmed as a pragmatic and sustainable countermeasure for cardioprotection, the precise underlying mechanisms for these beneficial effects remain to be defined.

One group of enzymes that is important in mediating the destructive effects of cardiovascular disease is the family of matrix metalloproteinases (MMPs). The MMPs are a large family of calcium-dependent, zinc-containing endopeptidases that have the ability to remodel the extracellular matrix in both physiological and pathological processes. Of this diverse family of enzymes, MMP-2 (also known as gelatinase A) is found in nearly all cell types and plays a key role in the cardiovascular system, ranging from heart development to ischemia/reperfusion (I/R) injury [3]. The release of MMP-2 increases with increasing duration of ischemia and correlates negatively with functional recovery. Over the past few decades, many approaches to the relationship between MMP-2 activity and infarct size have been studied [4]. These investigations have revealed that the inhibition of MMP-2 protects the heart from cardiac dysfunction [5]. However, the 
activation of MMP-2 during an ischemic insult is associated with a larger infarct size of the heart [6]. Myocardial ischemia followed by reperfusion results in $\mathrm{I} / \mathrm{R}$ injury characterized by a decreased myocardial function and tissue necrosis which is a marker of a long-term left ventricular function in patients who have suffered an acute myocardial infarction [7]. The purpose of this study was to assess the effects of 6 weeks of voluntary physical exercise on MMP-2 activity and release into the coronary effluent and to investigate the cardiac performance. We additionally measured the angina susceptibility of the heart, the basal blood pressure, and surviving aorta ring contraction and determined the cardiac infarct size after I/R-induced injury.

\section{Materials and Methods}

2.1. Animals and Experimental Design. All experimental procedures were performed in accordance with the standards of the European Community guidelines on the care and use of laboratory animals and had been approved by the local Institutional Ethics Committee at the University of Szeged.

Male Wistar rats $(n=70$, weighing 200-230 g; Toxi-Coop Zrt., Hungary) were randomly assigned to control and exercising groups. The exercising animals were placed individually into cages fitted with a running wheel (Acellabor Ltd., Budapest, Hungary) and were allowed free access to the wheel for $24 \mathrm{~h}$ per day for 6 weeks [8]. The exercising protocol, defined as a voluntary wheel-running model, was selected in an effort to isolate the effects of exercising from the additional stress associated with forced exercise protocols. During the exercising period, the average running distance was $3.91 \pm$ $1.27 \mathrm{~km} /$ day/animal. Control rats were placed in standard holding cages without a running wheel for the same period. All animals were housed in a temperature-controlled facility $\left(23^{\circ} \mathrm{C}\right)$ maintained on a 12:12 h light-dark cycle with food and water provided ad libitum.

2.2. Measurement of MMP-2 Activity. Metalloproteinase activity was detected by gelatin zymography. Sodium dodecyl sulfate-polyacrylamide gels (8\%) were copolymerized with $20 \mathrm{mg} / \mathrm{mL}$ gelatin. A constant amount of protein was loaded in each well under nonreducing conditions and was separated by electrophoresis. After electrophoresis, the gels were washed with $2.5 \%$ Triton X-100 and incubated overnight in incubation buffer $(50 \mathrm{mM}$ Tris- $\mathrm{HCl}, 150 \mathrm{mM}$ $\mathrm{NaCl}, 5 \mathrm{mM} \mathrm{CaCl}_{2}$, and $0.05 \% \mathrm{NaN}_{3}$ ) at $37^{\circ} \mathrm{C}$. After staining with $0.05 \%$ Coomassie Brilliant Blue in a mixture of methanol/acetic acid/water and being destained with aqueous $4 \%$ methanol/8\% acetic acid, enzyme activity was detected as colorless bands against the blue-stained background. A protein ladder (Spectra Multicolor Broad Range Protein Ladder, Thermo Scientific) was used to identify the individual enzymes (MMP-2, $72 \mathrm{kDa}$ and $64 \mathrm{kDa}$ isoforms) for molecular weights. Zymograms were scanned digitally and the band intensities were quantified by using Quantity One software (Bio-Rad, Hercules, CA, USA).

2.3. Measurement of Basal Blood Pressure. The animals were anaesthetized with $30.0 \%$ urethane $(0.50 \mathrm{~mL} / 100$ g, i.p. $)$ and the mean arterial blood pressure was measured in the right carotid artery, expressed as $\mathrm{mmHg}$. The right carotid artery was separated from the vagus nerve and clamped with a bulldog clamp for cannulation. Then the carotid artery cannulation was connected to the HAEMOSYS computerized complex hemodynamic analysis system (Experimetria UK, London) to record the mean blood pressure. After 10-15minute stabilization period, the baseline recording has carried out over a 10-minute period to examine the mean blood pressure. The core temperature of the rats was maintained at $37^{\circ} \mathrm{C}$ with a homeothermic control unit (Harvard Instrument, $\mathrm{UK})$.

2.4. Experimental Angina Provoked by Epinephrine Plus Phentolamine. The animals were anesthetized with $30.0 \%$ urethane $(0.50 \mathrm{~mL} / 100 \mathrm{~g}$, i.p. $)$ and the standard limb lead II of the surface electrocardiogram (ECG) was recorded by the HAEMOSYS system and expressed as $\mathrm{mV}$. The change in ST segment was measured and used as the index of angina severity. The mean ECG voltage $13 \mathrm{~ms}$ after the peak of the $\mathrm{S}$ wave was defined as the value of the ST segment, as described previously [9]. In the epinephrine plus phentolamine model, single doses of epinephrine $(10.0 \mu \mathrm{g} / \mathrm{kg})$ and $30 \mathrm{~s}$ later the $\alpha$ adrenoreceptor antagonist phentolamine $(15.0 \mathrm{mg} / \mathrm{kg})$ were administered into the tail vein of the rat. Each agent was dissolved in $0.20 \mathrm{~mL}$ of physiological saline and injected over $2 \mathrm{~s}$. To measure the difference in the amplitude of ST segment, the ECG recording has been carried out from the challenge of epinephrine over a 10-minute period.

2.5. Measurement of Arginine Vasopressin- (AVP-) Induced Heart Perfusion according to Langendorff. After cervical dislocation, the hearts were rapidly excised and then perfused according to Langendorff at a constant pressure of $75 \mathrm{mmHg}$ with Krebs-Henseleit buffer $(118.0 \mathrm{mM} \mathrm{NaCl}$, $4.70 \mathrm{mM} \mathrm{KCl}, 2.50 \mathrm{mM} \mathrm{CaCl}_{2}, 1.18 \mathrm{mM} \mathrm{MgSO}_{4}, 25.0 \mathrm{mM}$ $\mathrm{NaHCO}_{3}, 1.18 \mathrm{mM} \mathrm{KH} \mathrm{PO}_{4}$, and $5.50 \mathrm{mM}$ glucose), gassed with a mixture of $95 \% \mathrm{O}_{2} / 5 \% \mathrm{CO}_{2}$ at $37^{\circ} \mathrm{C}$. Following the cannulation, the heart perfusion in response to AVP was measured. The AVP quantity $(0.01,0.1$, and $1.0 \mu \mathrm{g})$ was added in Krebs-Henseleit buffer and then $0.5 \mathrm{~mL}$ of AVP-Krebs solution was injected into the Langendorff apparatus by way of a valve attached to the cannula. We administrated the perfusion changes every $30 \mathrm{~s}$ for $5 \mathrm{~min}$. Each dose of AVP was injected after a 15-minute stabilization period. Data are expressed as a percentage of the decrease relative to the basal value.

2.6. Measurement of Surviving Aorta Contraction. The rats were killed by cervical dislocation, and the abdominal aortas were removed and placed in chilled Krebs-Henseleit solution (118.0 mM NaCl, 4.70 mM KCl, $2.50 \mathrm{mM} \mathrm{CaCl}_{2}, 1.18 \mathrm{mM}$

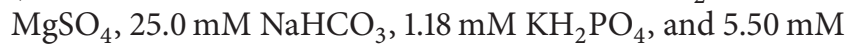
glucose) which was gassed with $95 \% \mathrm{O}_{2}$ and $5 \% \mathrm{CO}_{2}$. The aorta contraction was measured as described previously [10]. Briefly, the aortas were cleaned of all adipose and connective tissue, the abdominal region was cut into rings ( $3 \mathrm{~mm}$ long), and their weights were measured. To induce the contraction changes, we freshly added a $2 \mu \mathrm{g} / \mathrm{mL}$ dose of AVP into the 


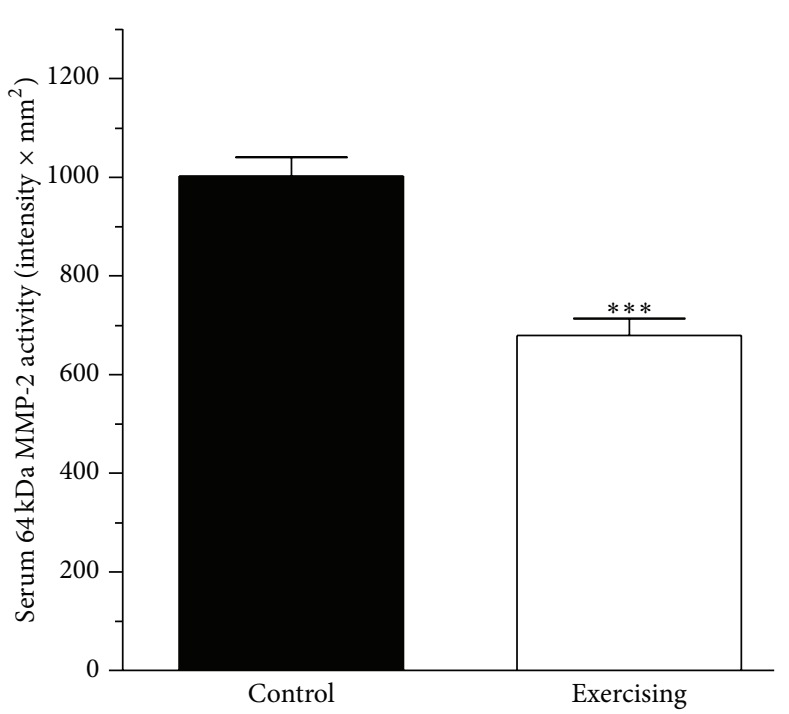

(a)

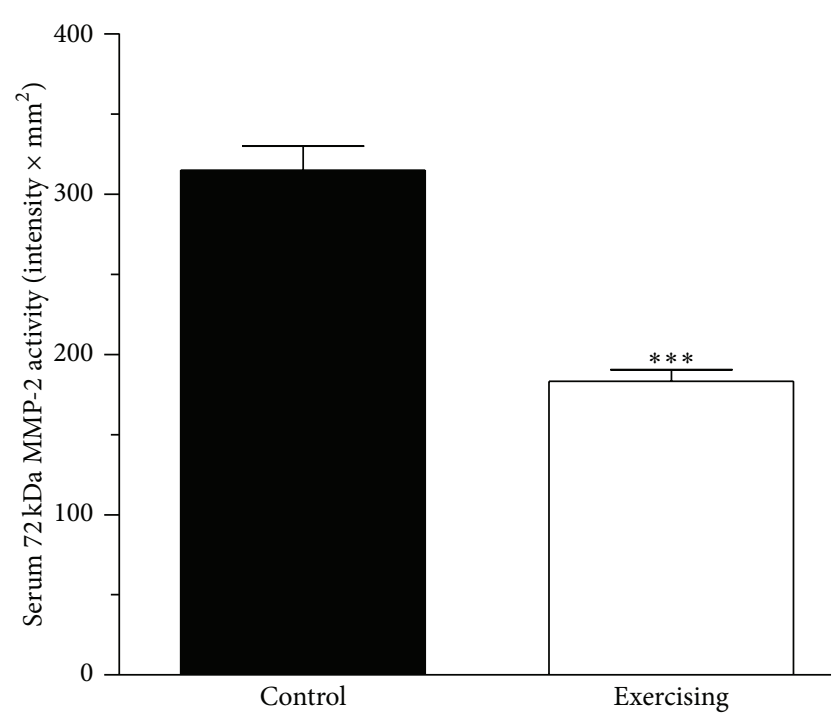

(b)

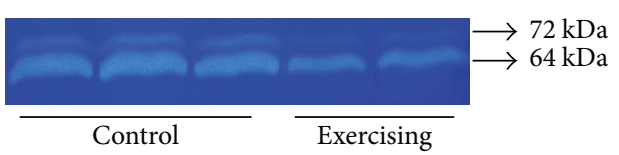

FIGURE 1: Serum matrix metalloproteinase-2 activity ( $64 \mathrm{kDa}$ and $72 \mathrm{kDa} \mathrm{MMP}-2$, expressed as intensity $\times$ mm $\left.{ }^{2}\right)$ in control ( $\left.\mathbf{}\right)$ and exercising (口) rats. Data are means \pm SEM, $n=12-13$. Statistical significance: ${ }^{* * *} P<0.001$ as compared with the control group. (a) Serum $64 \mathrm{kDa}$ matrix metalloproteinase-2 activity (64 kDa MMP-2, expressed as intensity $\times \mathrm{mm}^{2}$ ) in control ( $\square$ ) and exercising ( $\square$ ) rats. (b) Serum $72 \mathrm{kDa}$ matrix metalloproteinase-2 activity (72 kDa MMP-2, expressed as intensity $\left.\times \mathrm{mm}^{2}\right)$ in control ( $\left.\square\right)$ and exercising ( $\left.\square\right)$ rats. The representative image presents a zymographic picture.

incubation buffer [10]. The isometric tension was measured through the transducer, which was connected to an ISOSYS computerized program system (Experimetria, UK, London) for continuous recording of the blood vessel tension. The contractile response to vasopressin was expressed in terms of the tension of the aorta ring ( $\mathrm{g} / \mathrm{mg}$ ring weight).

2.7. Ischemia/Reperfusion Protocol. After cervical dislocation the hearts were rapidly excised and then perfused in Langendorff mode at a constant pressure of $75 \mathrm{mmHg}$ with KrebsHenseleit buffer, gassed with a mixture of $5 \% \mathrm{CO}_{2}+95 \% \mathrm{O}_{2}$ at $37^{\circ} \mathrm{C}$. After normoxic perfusion for $10 \mathrm{~min}$, local ischemia was induced by occlusion of the left anterior descending coronary artery (LAD) for $30 \mathrm{~min}$. This was followed by reperfusion for $120 \mathrm{~min}$. The coronary effluent was collected during the first $5 \mathrm{~min}$ of reperfusion and was concentrated in Amicon Ultra Centrifugal concentrating vessels $\left(5000 \mathrm{~g}, 4^{\circ} \mathrm{C}\right.$, Millipore, MA, USA) for MMP-2 activity determination via gelatin zymography. At the end of the protocol the hearts were stained with $1 \%$ Evans blue and then were frozen at $-20^{\circ} \mathrm{C}$ overnight.

2.8. Infarct Size Determination. Infarct size was measured after regional ischemia induced by LAD occlusion ex vivo [11]. Frozen hearts were cut into $2 \mathrm{~mm}$ thick cross-sectional slices. These slices were stained with 1\% 2,3,5-triphenyltetrazolium chloride (TTC) for $10 \mathrm{~min}$ at $37^{\circ} \mathrm{C}$. After TTC staining, the slices were transferred to a formalin (10\%) solution for $10 \mathrm{~min}$ and then placed in phosphate buffer $(\mathrm{pH}=6)$. Following this incubation, both sides of each slice were photographed with a digital camera. Infarct size was calculated as the percentage of the area at risk.

2.9. Statistics. The results shown in the figures are expressed as means \pm S.E.M. Differences between groups were determined with two-tailed Student's $t$-test and $P$ values less than 0.05 were considered significant.

\section{Results}

3.1. Sera $64 k D a$ and $72 k D a M M P-2$ Activity. Serum samples were collected from the lateral tail vein. We found that the 6-week voluntary physical exercise significantly $(P<0.001)$ decreased the serum MMP-2 activity (that of $64 \mathrm{kDa}$ MMP-2 from $1002.71 \pm 37.50$ to $679.73 \pm 34.35$ intensity $\times \mathrm{mm}^{2}$ and that of $72 \mathrm{kDa}$ MMP-2 from $314.93 \pm 14.80$ to $183.33 \pm 7.12$ intensity $\times \mathrm{mm}^{2}, n=12-13$ ). Data are shown in Figure 1 .

3.2. Measurement of Basal Blood Pressure. The basal blood pressure measured in the right carotid artery proved to be similar in the control and exercising rats (from $94.71 \pm 4.13$ to $91.48 \pm 1.69, n=14-18)$. Data are presented in Figure 2(a). 


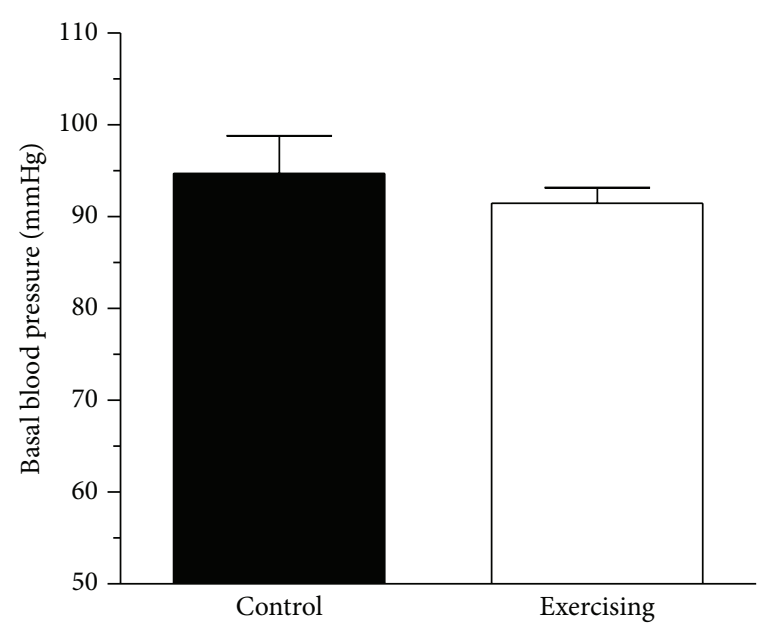

(a)

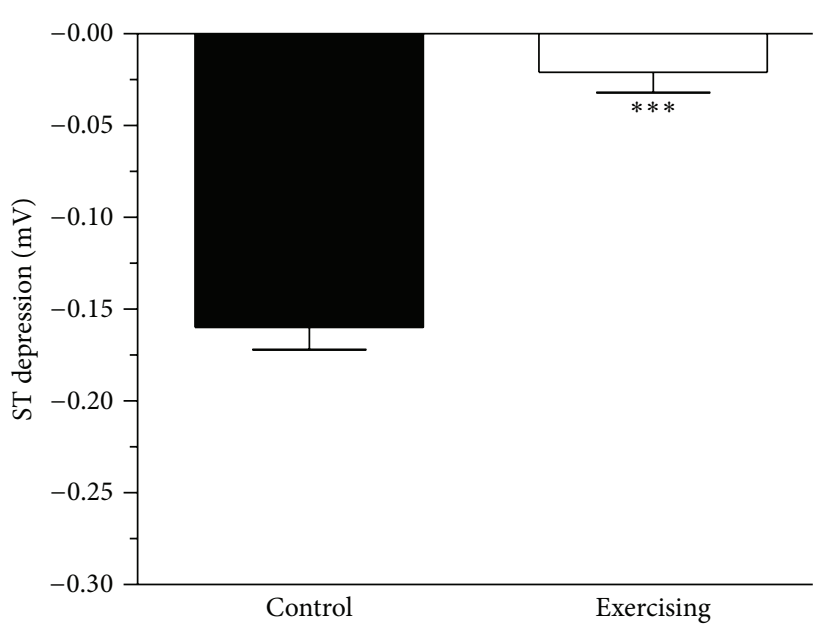

(b)

FIGURE 2: (a) The basal blood pressure ( $\mathrm{mmHg}$ ) in control ( $\mathbf{\square})$ and exercising ( $\square$ ) animals. Data are expressed as means \pm S.E.M., $n=14-18$. (b) The effect of recreational physical exercise on ST segment changes (measured in a lead II standard surface ECG; expressed in mV) following intravenous injection of epinephrine $(10.0 \mu \mathrm{g} / \mathrm{kg})$ and $30 \mathrm{~s}$ later phentolamine $(15.0 \mathrm{mg} / \mathrm{kg})$. Data are shown as means \pm S.E.M, $n=14-18$. Statistical significance: ${ }^{* * *} P<0.001$ as compared with the control group.

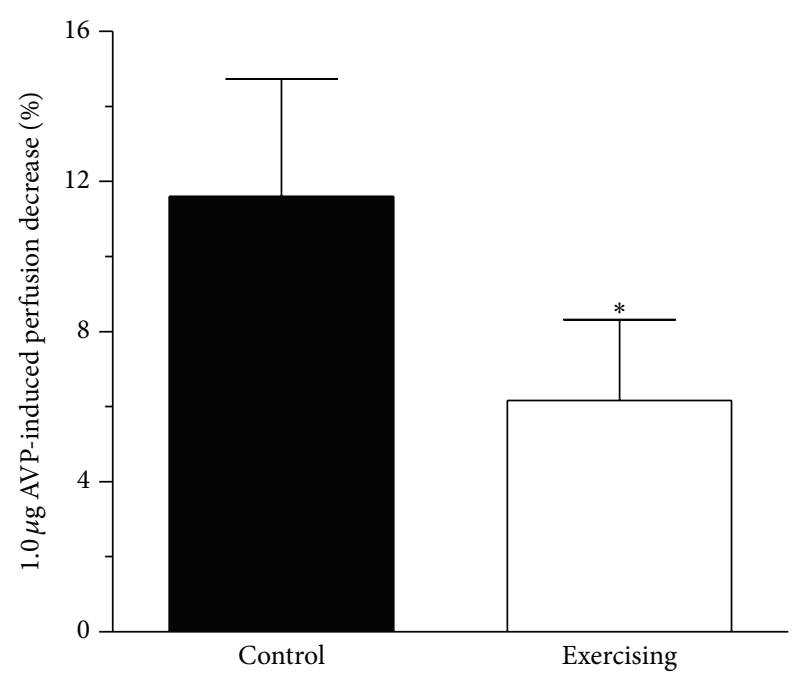

(a)

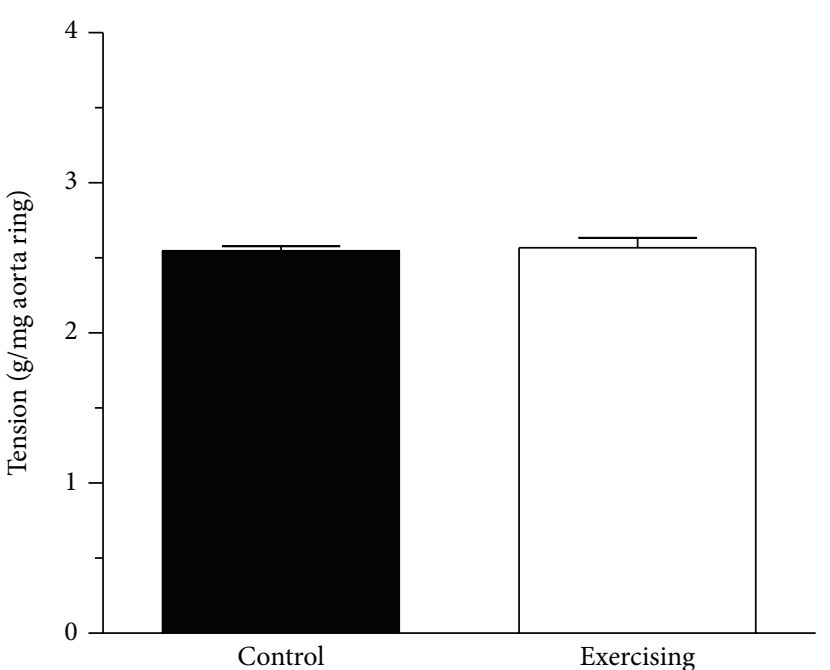

(b)

FIGURE 3: (a) The Langendorff heart perfusion decrease expressed as a percentage in response to $1.0 \mu \mathrm{g}$ arginine-vasopressin (AVP) in control $(\square)$ and exercising ( $\square$ ) rats. Means \pm S.E.M., $n=8-10$. Statistical significance: ${ }^{*} P<0.05$ as compared with the control group. (b) The effect of $2.0 \mu \mathrm{g}$ AVP-induced aorta ring contraction (expressed as g/mg aorta ring weight) on control ( $\mathbf{\square})$ and exercising ( $\square$ ) animals. Results are shown as means \pm S.E.M., $n=9-10$.

3.3. Experimental Angina Provoked by Epinephrine Plus Phentolamine. The administration of phentolamine $30 \mathrm{~s}$ after epinephrine caused a robust $(P<0.001)$ ST segment depression in the control rats $(-0.16 \pm 0.012 \mathrm{mV}, n=14-$ 18). As a result of 6 -week physical exercise we have found an improvement in ST segment changes $(-0.021 \pm 0.011 \mathrm{mV}$, $n=14-18, P<0.001)$. Data are shown in Figure $2(\mathrm{~b})$.

3.4. Measurement of Isolated Heart Perfusion Provoked by $A V P$. The perfusion measured according to Langendorff is illustrated in Figure 3(a). No differences in basal perfusion or in response to 0.01 or $0.1 \mu \mathrm{g}$ AVP were observed between the control and the exercising animals. However, 1.0 $\mu \mathrm{g}$ AVP revealed a significantly $(P<0.05)$ improved perfusion in the exercising group (from $11.6 \pm 3.14$ to $6.17 \pm 2.16 \%, n=8-10$ ).

3.5. Measurement of Aorta Contraction Provoked by AVP. Results observed in the experiment involving the surviving aorta ring contraction are demonstrated in Figure 3(b). In response to the $2.0 \mu \mathrm{g} / \mathrm{mL}$ dose of AVP, we found no significant difference between the control and the exercising 


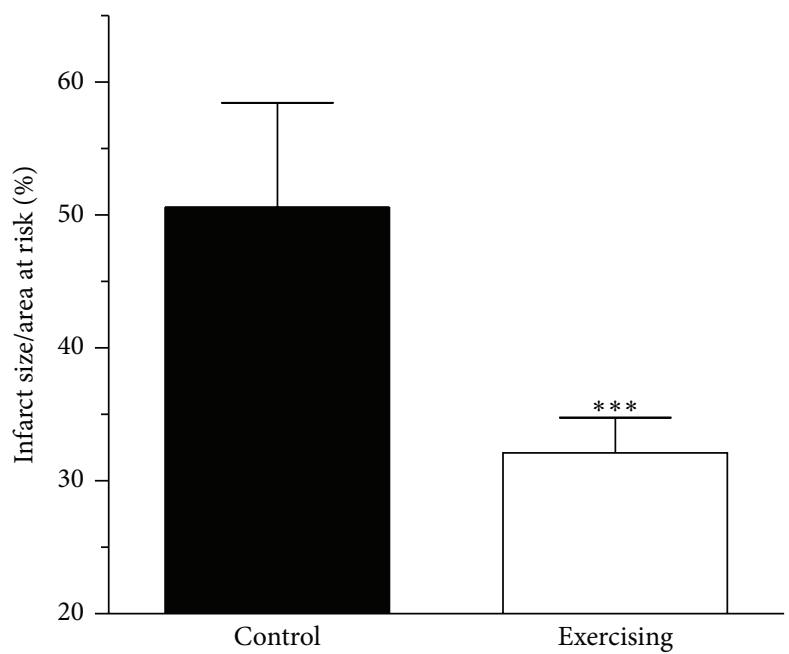

(a)

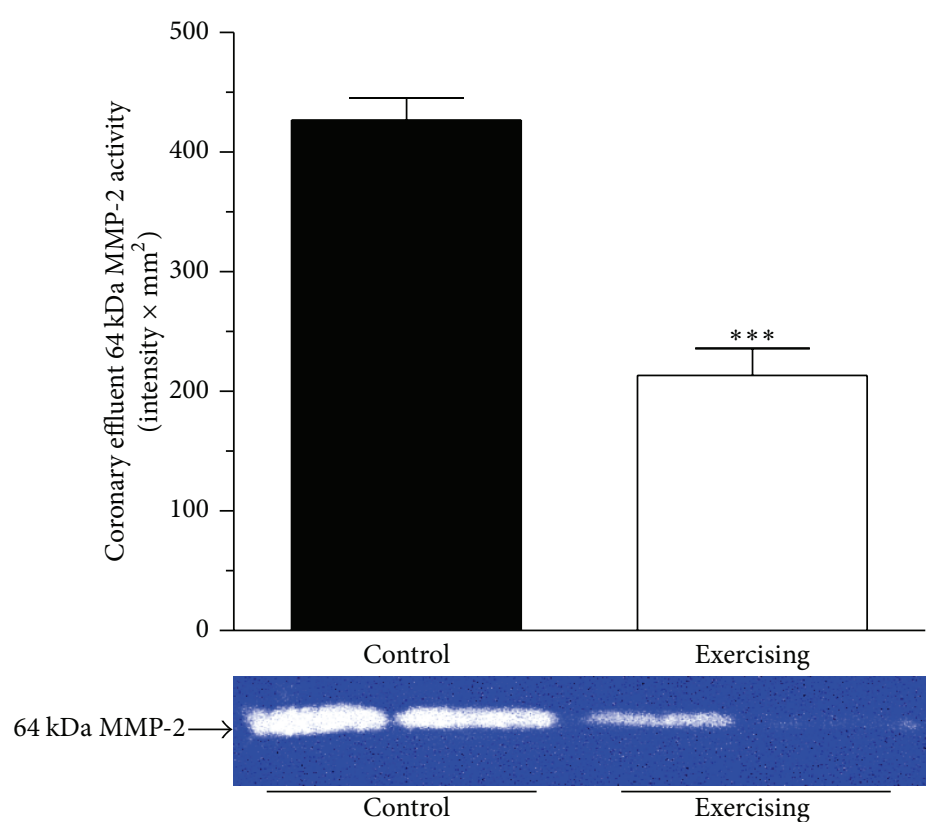

(b)

FIGURE 4: (a) Infarct size is demonstrated as a percentage of the area at risk in control ( $\square$ ) and exercising ( $\square$ ) groups. Means \pm S.E.M., $n=11$ 12. Statistical significance: ${ }^{* * *} P<0.001$ as compared with the control animals. (b) Densitometrically assessed MMP-2 activity in coronary effluent collected from isolated perfused hearts during the first $5 \mathrm{~min}$ of reperfusion $\left(64 \mathrm{kDa}\right.$ MMP-2, expressed as intensity $\left.\times \mathrm{mm}^{2}\right)$ in control $(\square)$ and exercising $(\square)$ rats. Means \pm S.E.M., $n=10$. Statistical significance: ${ }^{* * *} P<0.001$ as compared with the control group.

animals (from $2.55 \pm 0.03$ to $2.57 \pm 0.07 \mathrm{~g} / \mathrm{mg}$ aorta ring, $n=9-10)$.

3.6. Determination of Infarct Size. Figure 4(a) shows the infarct size after $30 \mathrm{~min}$ of LAD occlusion and $120 \mathrm{~min}$ of reperfusion expressed as a percentage of the area at risk. 6 weeks of voluntary exercise training significantly $(P<0.001)$ reduced the infarct size in the exercising animals as compared with the control group (from $50.6 \pm 7.86 \%$ to $32.12 \pm 2.66 \%$, $n=11-12)$.

3.7. Coronary Effluent MMP-2 Activity. The coronary effluent MMP-2 activity was determined after the 30-minute LAD occlusion, during the first $5 \mathrm{~min}$ of reperfusion. In the exercising group the activity of the $64 \mathrm{kDa}$ MMP-2 isoform was significantly $(P<0.001)$ decreased from $426.94 \pm 18.37$ to $213.32 \pm 22.45$ intensity $\times \mathrm{mm}^{2}, n=10$ ). Data are presented in Figure 4(b).

\section{Discussion}

We demonstrated that 6 weeks of voluntary exercise was able to decrease the levels of serum and coronary effluent MMP-2 activity, reduce the myocardial infarct size, and improve the angina susceptibility of the heart. Voluntary wheel-running additionally resulted in an improvement in myocardial perfusion.

We used a voluntary wheel-running model where the animals were able to self-select the time, duration, and intensity of exercise in a nonstressful environment. Previously, 3-4 weeks of voluntary wheel-running had been demonstrated to induce robust physiological hypertrophy, which provokes a beneficial adaptive response of the cardiovascular system $[12,13]$. We therefore employed a 6 -week exercise protocol to provide sufficient stimuli for adaptation.

Regular physical exercise results in physiological left ventricular hypertrophy which contributes to several cardiovascular benefits. Resting bradycardia has been considered to be the hallmark cardiovascular effect of exercise-training adaptation $[14,15]$. Changes after regular exercise might result in an improvement in bioenergetics and metabolic status and modification in endogenous defense system. There is a broad consensus that antioxidative mechanisms play a key role in cardioprotection through training-induced upregulation $[16,17]$. However, besides the antioxidant defenses, another mechanism, MMP-2 secretion, can be modulated by exercise. Most previous studies have reported data concerning MMP activity after various training models in either the skeletal muscle [18] or the cardiac tissues [19]. Less information is available concerning the influence of exercise-induced MMP2 in the circulation. To investigate this question, serum and released MMP-2 activity into the coronary effluent were analyzed. Urso et al. measured the serum MMP-2 activity after a single bout of exercise but did not find a change in their study [20]. In our present investigation involving wheelrunning animals, we hypothesized that exercise would influence the serum MMP-2 activation. Our zymography analyses revealed that 6 weeks of voluntary exercise training decreased 
the serum levels of the 64 - and $72 \mathrm{kDa}$ MMP- 2 forms in the exercising animals as compared with the control group. Previous human studies have demonstrated that physical exercise can affect the levels of MMPs following acute and chronic interventions. Under coronary risk events, the elevations of MMP-2 and MMP-9 are correlated with increased inflammation [21]. The decrease in MMPs could be related to expression of the tissue inhibitors of metalloproteinases (TIMPs), protease degradation, and reduction of proinflammatory cytokine tumor necrosis factor-alpha (TNF- $\alpha$ ) [22]. The lowering levels of inflammatory biomarkers mediate the inhibitory effect of exercise on MMP-2 and MMP-9 levels [23]. Lucotti et al. have investigated the effects of aerobic exercise and found that the exercise program caused about $20 \%$ reduction in TNF- $\alpha$ and MMP-2 levels [24]. Thus, the alterations on MMP's circulating concentrations might be a good reflection of the exercise effect on inflammation markers. The decrease in the level of serum MMP-2 is a systemic effect which might be contributing to the multiple adaptation mechanisms of physical exercise.

It is well known that the increased activity of MMP-2 during cardiac $\mathrm{I} / \mathrm{R}$ contributes to the disruption of the endothelial layer and increase of its secretion into the coronary circulation. Cheung et al. first reported that MMP-2 can be released into the coronary effluent of perfused rat hearts. The release of MMP-2 peaked during the first and fifth minutes of reperfusion and was enhanced with increasing duration of ischemia [25]. In a previous study, Lalu et al. examined the effect of I/R on the gelatinolytic activity of MMP-2 in the heart ventricles and coronary effluent. Gelatinolytic activities of MMP-2 were detected before and after I/R and it was found that the release of MMP-2 into the coronary effluent was higher after I/R. It is known that in the first minutes of reperfusion there is a burst of reactive oxygen species (ROS) which determine the severity of the reperfusion injury [26]. The release of MMP-2 correlates negatively with the functional recovery [27]. Our data demonstrated that 6-week voluntary exercise was protective against reperfusion damage, since we found that the release of $64 \mathrm{kDa}$ MMP-2 into the perfusate decreased significantly in the exercising rats. However, we did not identify the $72 \mathrm{kDa}$ isoform activity in the coronary effluent.

Increased activity of the MMP-2 into the perfusate contributes to the disruption of the endothelial layer and has a negative impact on the vascular permeability and leads to coronary artery disease and heart failure [28]. However, the decrease in infarct size leads to an improved cardiac function [29-33]. Human epidemiological data clearly suggest that regular exercise reduces the risk of death during clinical I/R injury [34]. The results of our study show that 6-week voluntary wheel-running provides protection against I/R injury by reducing the myocardial infarct size. The extent of the necrotic area in exercised hearts was lessened by more than $50 \%$. Doustar et al. found that 4 -week resistance training did not protect the heart against I/R-induced injury [35]. The difference in these results could have resulted from methodological differences, such as the type and duration of exercise. The relationship between MMP-2 and infarct size was supported in a large body of different experimental evidences [4, 36, 37]. Our current data support this possible link between released MMP-2 activity and reduced infarct size.

That exercise training resulting in cardiovascular benefits that causes functional and structural adaptations is widely investigated. During exercise, increased blood flow and shear stress augment endothelium dependent vasodilatation through the upregulation of endothelial nitric oxide synthase (eNOS) [28]. The nitric oxide (NO), particularly derived from eNOS, has been implicated in the cardioprotection offered by exercise [38]. Increased arterial dilation induced by NO improves myocardial oxygen supply [39] and may indicate additional endothelium-dependent functions that prevent ischemic events. Exercise-induced NO may be a potential inducer of heme oxygenase (HO) enzyme system. In earlier investigations, many researches demonstrate the beneficial roles of the $\mathrm{HO}$ enzyme system in cardiovascular function $[10,40,41]$. In a previous study, Sun et al. found that exercise-induced elevation of vascular $\mathrm{HO}$ and enhanced $\mathrm{HO}$-related dilation demonstrate the direct participation of the $\mathrm{HO}$ system in cardiovascular adaptation [42]. In a diet-induced obese mouse model, Hafstad et al. showed that the impairment of left ventricular (LV) function and mechanoenergetics were normalized by moderate-intensity training. They demonstrated that the changes were associated with altered myocardial substrate utilization and improved mitochondrial capacity as well as reduced oxidative stress, fibrosis, and intracellular matrix metalloproteinase- 2 content [43].

To prove the adaptive and protective effects of voluntary wheel-running exercise, cardiac parameters were measured in this study. We detected the ST segment depression, which is considered a reliable ECG finding and has been associated with a worse prognosis for patients with coronary artery disease [44]. 6 weeks of voluntary exercise diminished the ST segment depression and therefore improved the ischemia susceptibility of the heart.

We have observed that basal normoxic heart perfusion was similar in the exercising group and the control group. We therefore utilized AVP, which can regulate the hemodynamic parameters by inducing moderate vasoconstriction. In response to $1.0 \mu \mathrm{g}$ AVP, a significant improvement in heart perfusion was observed after exercise training. The AVPinduced perfusion changes imply that a 6-week period of voluntary exercise may be effective in producing functional and structural adaptations in the cardiovascular system.

We also measured the AVP-induced aorta ring contraction and the basal blood pressure. In our earlier study, the surviving aorta ring contraction was measured by incubation with $\operatorname{AVP}(2.0 \mu \mathrm{g} / \mathrm{mL})$ in male and female rats [10]. The $2.0 \mu \mathrm{g} / \mathrm{mL}$ AVP dose was earlier the most effective one for the detection of gender differences, so we used this treatment in the present investigation [10]. Despite our results revealing favorable adaptation in the cardiovascular system after voluntary exercise training, no differences in aorta contraction were observed between the control and the exercising animals.

Similarly, no alteration in basal blood pressure was detected between the control and the exercising rats. In 
another investigation, Roque et al. measured the hemodynamic parameters after swimming training in normotensive rats and also found no blood pressure changes after exercise [45]. The effect of aerobic exercise training on the blood pressure of normotensive animals and humans seems to be minimal and our results are in agreement with those of other studies. Other investigations clearly demonstrated that hypertensive rats and rats with metabolic syndrome had higher blood pressure levels than did normotensive rats in which aerobic exercise did not induce any change in the blood pressure $[46,47]$.

In this current study, wheel-running exercising decreased the activities of both $64 \mathrm{kDa}$ and $72 \mathrm{kDa}$ MMP-2 in the serum and also the release of MMP-2 from the heart into the coronary effluent as a consequence of 30-minute LAD occlusion. Similar to the decreases in the MMP-2 values, the infarct size was also reduced. Furthermore, such a training period seems to be a potent stimulus for functional recovery in respect of the myocardial perfusion and ischemic susceptibility of the heart.

In conclusion, our results show adaptive and cardioprotective effects of voluntary wheel-running exercise. The reduced activity of serum MMP-2 might be contributing to the multiple adaptation mechanisms of 6-week physical exercise. The fact that the infarct size is decreased suggests that liberation of MMP-2 into the perfusate could be part of cardioprotective effects. Moreover, our training program was able to improve the angina susceptibility of the heart and causes functional recovery detected by AVP-induced perfusion changes.

\section{Conflict of Interests}

The authors declare that there is no conflict of interests in relation to this work.

\section{Authors' Contribution}

All authors participated in the design and interpretation of the studies in the analysis of the data and in the drafting and review of the paper. Renáta Szabó and Anikó Pósa contributed equally to this paper as first authors.

\section{Acknowledgments}

This research was realized in the frames of TÁMOP 4.2.4. A/2-11-1-2012-0001 "National Excellence Program: Elaborating and Operating an Inland Student and Researcher Personal Support System." The project was subsidized by the European Union and cofinanced by the European Social Fund.

\section{References}

[1] S. K. Agarwal, "Cardiovascular benefits of exercise," International Journal of General Medicine, vol. 5, pp. 541-545, 2012.

[2] P. Kokkinos, "Physical activity, health benefits, and mortality risk," ISRN Cardiology, vol. 2012, Article ID 718789, 14 pages, 2012.
[3] A. D. Kandasamy, A. K. Chow, M. A. M. Ali, and R. Schulz, "Matrix metalloproteinase-2 and myocardial oxidative stress injury: beyond the matrix," Cardiovascular Research, vol. 85, no. 3, pp. 413-423, 2010.

[4] M. Donato, V. D’Annunzio, B. Buchholz et al., "Role of matrix metalloproteinase-2 in the cardioprotective effect of ischaemic postconditioning," Experimental Physiology, vol. 95, no. 2, pp. 274-281, 2010.

[5] J. Fert-Bober, H. Leon, J. Sawicka et al., "Inhibiting matrix metalloproteinase-2 reduces protein release into coronary effluent from isolated rat hearts during ischemia-reperfusion," Basic Research in Cardiology, vol. 103, no. 5, pp. 431-443, 2008.

[6] W. Wang, G. Sawicki, and R. Schulz, "Peroxynitrite-induced myocardial injury is mediated through matrix metalloproteinase-2," Cardiovascular Research, vol. 53, no. 1, pp. 165-174, 2002.

[7] T. Baks, R.-J. Van Geuns, E. Biagini et al., "Recovery of left ventricular function after primary angioplasty for acute myocardial infarction," European Heart Journal, vol. 26, no. 11, pp. 1070-1077, 2005.

[8] Z. Szalai, A. Szász, I. Nagy et al., "Anti-inflammatory effect of recreational exercise in TNBS-Induced colitis in rats: role of NOS/HO/MPO system," Oxidative Medicine and Cellular Longevity, vol. 2014, Article ID 925981, 11 pages, 2014.

[9] J. Nemcsik, É. Morschl, J. Egresits et al., "Raloxifene lowers ischaemia susceptibility by increasing nitric oxide generation in the heart of ovariectomized rats in vivo," European Journal of Pharmacology, vol. 495, no. 2-3, pp. 179-184, 2004.

[10] A. Pósa, K. Kupai, R. Ménesi et al., "Sexual dimorphism of cardiovascular ischemia susceptibility is mediated by heme oxygenase," Oxidative Medicine and Cellular Longevity, vol. 2013, Article ID 521563, 11 pages, 2013.

[11] K. Kupai, C. Csonka, V. Fekete et al., "Cholesterol dietinduced hyperlipidemia impairs the cardioprotective effect of postconditioning: role of peroxynitrite," American Journal of Physiology-Heart and Circulatory Physiology, vol. 297, no. 5, pp. H1729-H1735, 2009.

[12] Y. Wang, U. Wisloff, and O. J. Kemi, "Animal models in the study of exercise-induced cardiac hypertrophy," Physiological Research, vol. 59, no. 5, pp. 633-644, 2010.

[13] J. P. Konhilas, A. H. Maass, S. W. Luckey, B. L. Stauffer, E. N. Olson, and L. A. Leinwand, "Sex modifies exercise and cardiac adaptation in mice," American Journal of Physiology: Heart and Circulatory Physiology, vol. 287, no. 6, pp. H2768-H2776, 2004.

[14] A. D. Harthmann, K. de Angelis, L. P. Costa et al., "Exercise training improves arterial baro- and chemoreflex in control and diabetic rats," Autonomic Neuroscience: Basic and Clinical, vol. 133, no. 2, pp. 115-120, 2007.

[15] A. Medeiros, E. M. Oliveira, R. Gianolla, D. E. Casarini, C. E. Negrão, and P. C. Brum, "Swimming training increases cardiac vagal activity and induces cardiac hypertrophy in rats," Brazilian Journal of Medical and Biological Research, vol. 37, no. 12, pp. 1909-1917, 2004.

[16] K. L. Hamilton, J. C. Quindry, J. P. French et al., "MnSOD antisense treatment and exercise-induced protection against arrhythmias," Free Radical Biology \& Medicine, vol. 37, no. 9, pp. 1360-1368, 2004.

[17] K. L. Hamilton, J. L. Staib, T. Phillips, A. Hess, S. L. Lennon, and S. K. Powers, "Exercise, antioxidants, and HSP72: protection 
against myocardial ischemia/reperfusion," Free Radical Biology and Medicine, vol. 34, no. 7, pp. 800-809, 2003.

[18] E. Rullman, J. Norrbom, A. Strömberg et al., "Endurance exercise activates matrix metalloproteinases in human skeletal muscle," Journal of Applied Physiology, vol. 106, no. 3, pp. 804-812, 2009.

[19] M. Bellafiore, G. Battaglia, A. Bianco, F. Farina, A. Palma, and A. Paoli, "The involvement of MMP-2 and MMP-9 in heart exercise-related angiogenesis," Journal of Translational Medicine, vol. 11, no. 1, article 283, 2013.

[20] M. L. Urso, J. R. Pierce, J. A. Alemany, E. A. Harman, and B. C. Nindl, "Effects of exercise training on the matrix metalloprotease response to acute exercise," European Journal of Applied Physiology, vol. 106, no. 5, pp. 655-663, 2009.

[21] D. da Cunha Nascimento, R. de Cassia Marqueti Durigan, R. A. Tibana, J. L. Durigan, J. W. Navalta, and J. Prestes, "The response of matrix metalloproteinase-9 and -2 to exercise," Sports Medicine. In press.

[22] A. Niessner, B. Richter, M. Penka et al., "Endurance training reduces circulating inflammatory markers in persons at risk of coronary events: Impact on plaque stabilization?" Atherosclerosis, vol. 186, no. 1, pp. 160-165, 2006.

[23] H. B. Kwak, "Aging, exercise, and extracellular matrix in the heart," Journal of Exercise Rehabilitation, vol. 9, no. 3, pp. 338347, 2013.

[24] P. Lucotti, L. D. Monti, E. Setola et al., "Aerobic and resistance training effects compared to aerobic training alone in obese type 2 diabetic patients on diet treatment," Diabetes Research and Clinical Practice, vol. 94, no. 3, pp. 395-403, 2011.

[25] P.-Y. Cheung, G. Sawicki, M. Wozniak, W. Wang, M. W. Radomski, and R. Schulz, "Matrix metalloproteinase-2 contributes to ischemia-reperfusion injury in the heart," Circulation, vol. 101, no. 15, pp. 1833-1839, 2000.

[26] M. M. Lalu, C. Csonka, Z. Giricz, T. Csont, R. Schulz, and P. Ferdinandy, "Preconditioning decreases ischemia/reperfusioninduced release and activation of matrix metalloproteinase-2," Biochemical and Biophysical Research Communications, vol. 296, no. 4, pp. 937-941, 2002.

[27] R. Schulz, "Intracellular targets of matrix metalloproteinase-2 in cardiac disease: rationale and therapeutic approaches," Annual Review of Pharmacology and Toxicology, vol. 47, pp. 211-242, 2007.

[28] S. Gielen, G. Schuler, and V. Adams, "Cardiovascular effects of exercise training: molecular mechanisms," Circulation, vol. 122, no. 12, pp. 1221-1238, 2010.

[29] B. Juhasz, P. Der, P. Szodoray et al., "Adrenocorticotrope hormone fragment (4-10) attenuates the ischemia/reperfusioninduced cardiac injury in isolated rat hearts," Antioxidants and Redox Signaling, vol. 9, no. 11, pp. 1851-1861, 2007.

[30] B. Juhasz, P. Der, T. Turoczi, I. Bacskay, E. Varga, and A. Tosaki, "Preconditioning in intact and previously diseased myocardium: laboratory or clinical dilemma?" Antioxidants and Redox Signaling, vol. 6, no. 2, pp. 325-333, 2004.

[31] B. Juhasz, A. Kertész, J. Balla et al., "Cardioprotective effects of sour cherry seed extract (SCSE) on the hypercholesterolemic rabbit heart," Current Pharmaceutical Design, vol. 19, no. 39, pp. 6896-6905, 2013.

[32] B. Juhasz, B. Varga, A. Czompa et al., "Postischemic cardiac recovery in heme oxygenase-1 transgenic ischemic/reperfused mouse myocardium," Journal of Cellular and Molecular Medicine, vol. 15, no. 9, pp. 1973-1982, 2011.

[33] A. Kertész, M. Bombicz, D. Priksz et al., "Adverse impact of dietinduced hypercholesterolemia on cardiovascular tissue homeostasis in a rabbit model: time-dependent changes in cardiac parameters," International Journal of Molecular Sciences, vol. 14, no. 9, pp. 19086-19108, 2013.

[34] J. L. V. Reeve, A. M. Duffy, T. O’Brien, and A. Samali, "Don't lose heart-therapeutic value of apoptosis prevention in the treatment of cardiovascular disease," Journal of Cellular and Molecular Medicine, vol. 9, no. 3, pp. 609-622, 2005.

[35] Y. Doustar, F. G. Soufi, A. Jafary, M. M. Saber, and R. Ghiassie, "Role of four-week resistance exercise in preserving the heart against ischaemia-reperfusion-induced injury," Cardiovascular Journal of Africa, vol. 23, no. 8, pp. 451-455, 2012.

[36] Z. Giricz, M. M. Lalu, C. Csonka, P. Bencsik, R. Schulz, and P. Ferdinandy, "Hyperlipidemia attenuates the infarct sizelimiting effect of ischemic preconditioning: role of matrix metalloproteinase-2 inhibition," Journal of Pharmacology and Experimental Therapeutics, vol. 316, no. 1, pp. 154-161, 2006.

[37] V. D’Annunzio, M. Donato, L. Erni et al., "Rosuvastatin given during reperfusion decreases infarct size and inhibits matrix metalloproteinase- 2 activity in normocholesterolemic and hypercholesterolemic rabbits," Journal of Cardiovascular Pharmacology, vol. 53, no. 2, pp. 137-144, 2009.

[38] D. B. Thorp, J. V. Haist, J. Leppard, K. J. Milne, M. Karmazyn, and E. G. Noble, "Exercise training improves myocardial tolerance to ischemia in male but not in female rats," American Journal of Physiology-Regulatory Integrative and Comparative Physiology, vol. 293, no. 1, pp. R363-R371, 2007.

[39] R. Hambrecht, E. Fiehn, C. Weigl et al., "Regular physical exercise corrects endothelial dysfunction and improves exercise capacity in patients with chronic heart failure," Circulation, vol. 98, no. 24, pp. 2709-2715, 1998.

[40] D. D. Haines, I. Lekli, P. Teissier, I. Bak, and A. Tosaki, "Role of haeme oxygenase-1 in resolution of oxidative stress-related pathologies: focus on cardiovascular, lung, neurological and kidney disorders," Acta Physiologica, vol. 204, no. 4, pp. 487501, 2012.

[41] A. Czompa, A. Gyongyosi, A. Czegledi et al., "Cardioprotection afforded by sour cherry seed kernel: the role of heme oxygenase1," Journal of Cardiovascular Pharmacology, vol. 64, no. 5, pp. 412-419, 2014.

[42] M.-W. Sun, M.-F. Zhong, J. Gu, F.-L. Qian, J.-Z. Gu, and H. Chen, "Effects of different levels of exercise volume on endothelium-dependent vasodilation: roles of nitric oxide synthase and heme oxygenase," Hypertension Research, vol. 31, no. 4, pp. 805-816, 2008.

[43] A. D. Hafstad, J. Lund, E. Hadler-Olsen, A. C. Höper, T. S. Larsen, and E. Aasum, "High- And moderate-Intensity training normalizes ventricular function and mechanoenergetics in mice with diet-Induced obesity," Diabetes, vol. 62, no. 7, pp. 2287-2294, 2013.

[44] G. A. Lanza, M. Mustilli, A. Sestito, F. Infusino, G. A. Sgueglia, and F. Crea, "Diagnostic and prognostic value of ST segment depression limited to the recovery phase of exercise stress test," Heart, vol. 90, no. 12, pp. 1417-1421, 2004.

[45] F. R. Roque, U. P. R. Soci, K. de Angelis et al., "Moderate exercise training promotes adaptations in coronary blood flow 
and adenosine production in normotensive rats," Clinics, vol. 66, no. 12, pp. 2105-2111, 2011.

[46] P. W. Caponi, A. M. Lehnen, G. H. Pinto et al., "Aerobic exercise training induces metabolic benefits in rats with metabolic syndrome independent of dietary changes," Clinics, vol. 68, no. 7, pp. 1010-1017, 2013.

[47] N. M. Leguisamo, A. M. Lehnen, U. F. Machado et al., "GLUT4 content decreases along with insulin resistance and high levels of inflammatory markers in rats with metabolic syndrome," Cardiovascular Diabetology, vol. 11, article 100, 2012. 


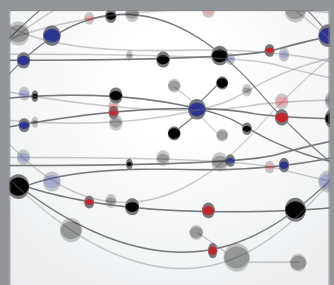

The Scientific World Journal
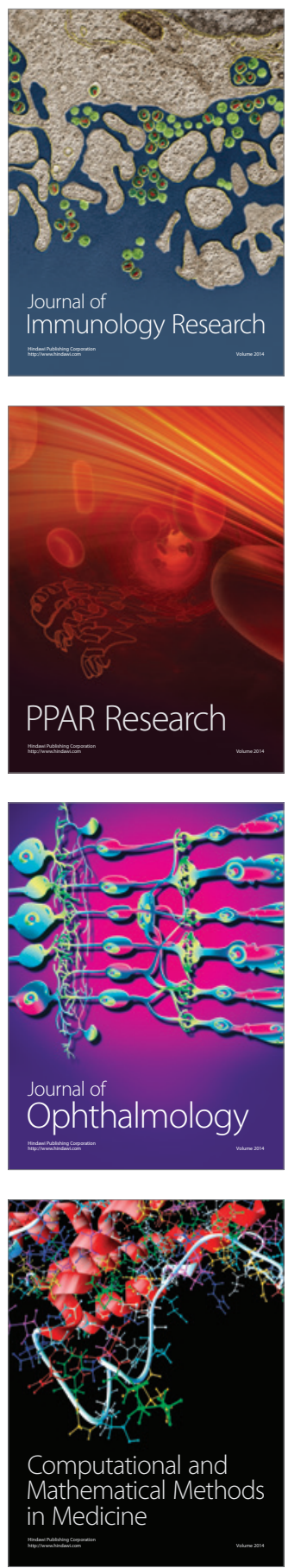

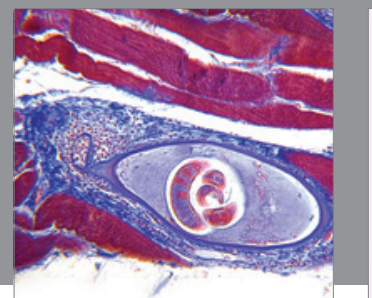

Gastroenterology

Research and Practice
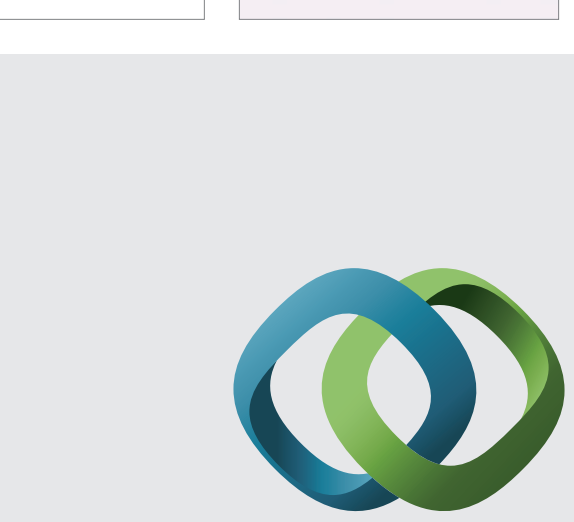

\section{Hindawi}

Submit your manuscripts at

http://www.hindawi.com
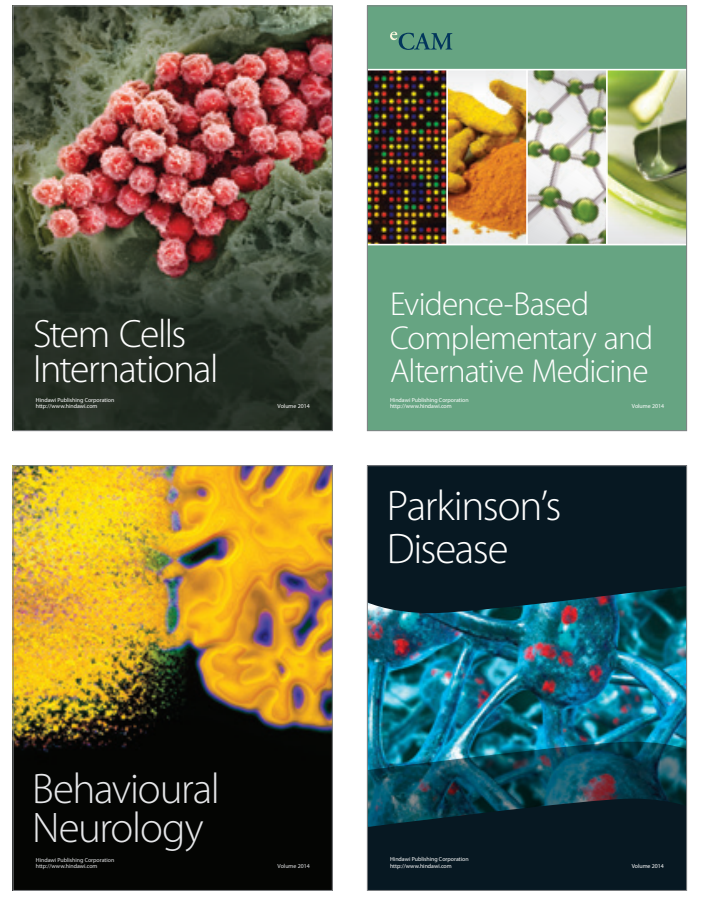
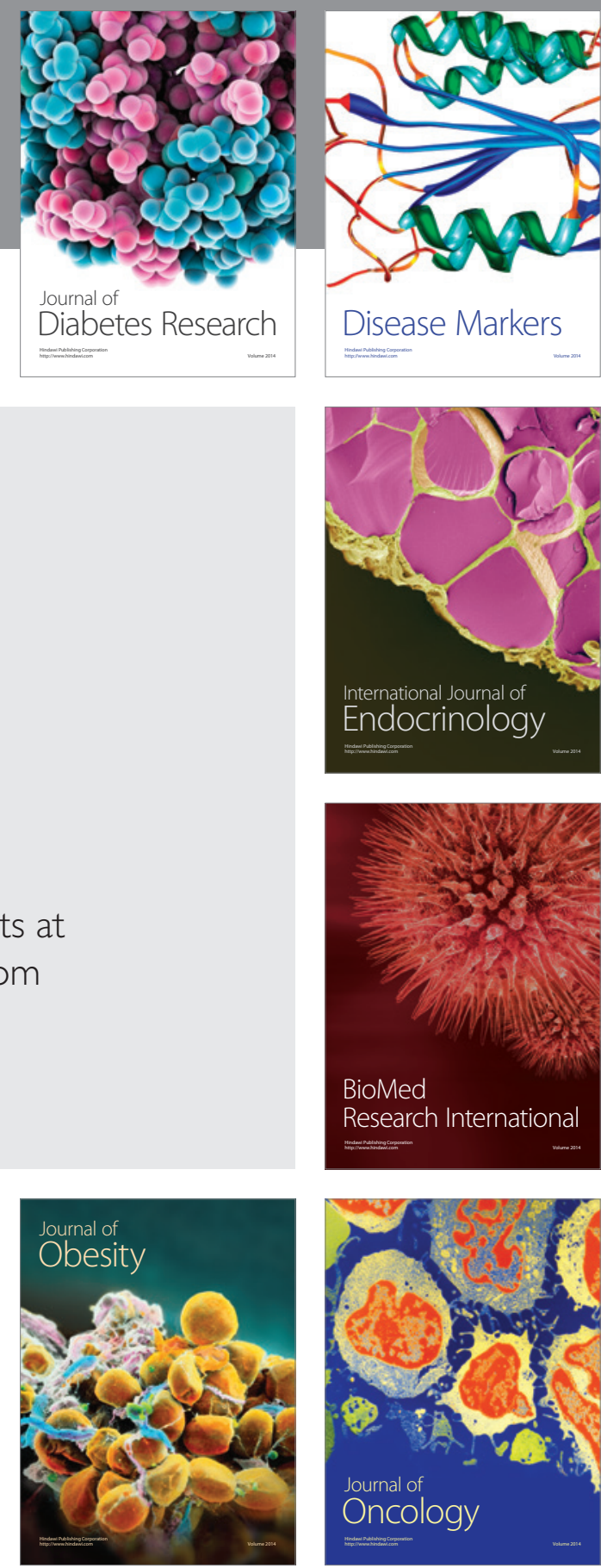

Disease Markers
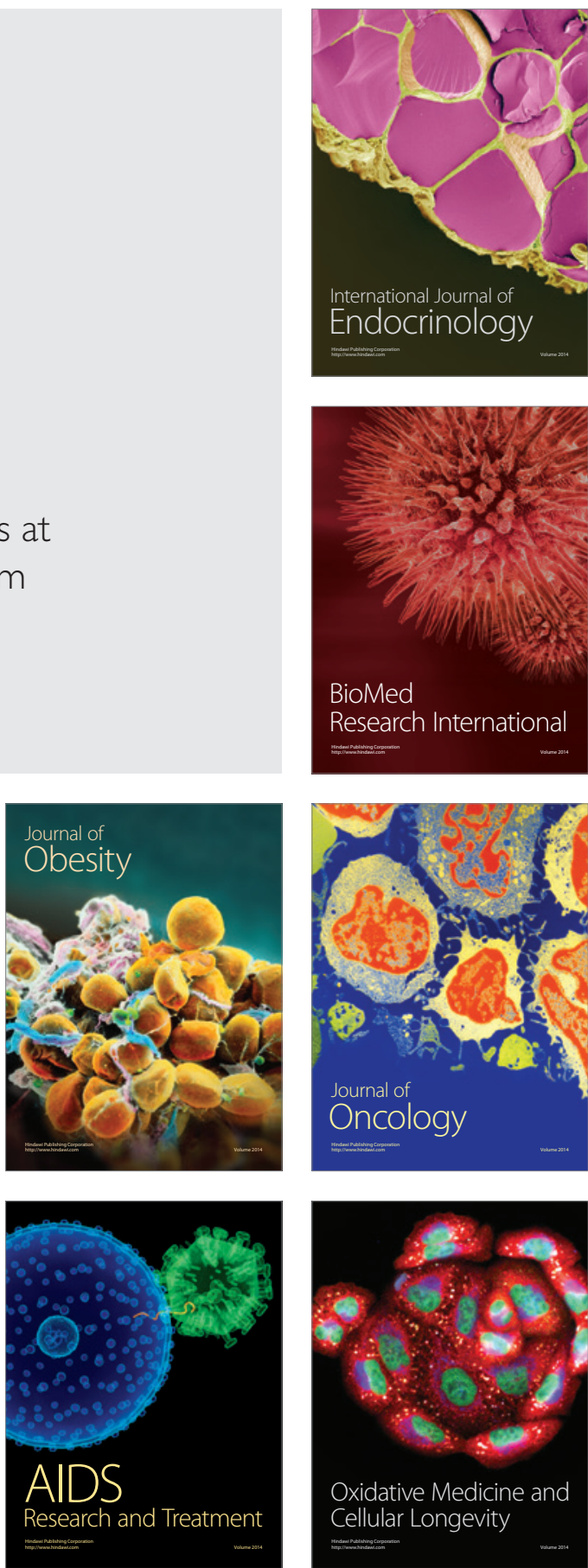